\title{
Overexpressed proprotein convertase $1 / 3$ induces an epithelial-mesenchymal transition in airway epithelium
}

\author{
Sang-Nam Lee ${ }^{1}$, Da-Hyung Lee ${ }^{1}$, Myung Hyun Sohn ${ }^{2,3,4}$ and Joo-Heon Yoon 1,2,5,6 \\ Affiliations: ${ }^{1}$ Research Centre for Natural Human Defence System, Yonsei University College of Medicine, \\ Seoul, ${ }^{2}$ BK 21 Project for Medical Science, Yonsei University College of Medicine, Seoul, ${ }^{3}$ Dept of Paediatrics \\ and Institute of Allergy, Yonsei University College of Medicine, Seoul, ${ }^{4}$ Severance Biomedical Science Institute, \\ Yonsei University College of Medicine, Seoul, ${ }^{5}$ Dept of Otorhinolaryngology, Yonsei University College of \\ Medicine, Seoul, ${ }^{6}$ The Airway Mucus Institute, Yonsei University College of Medicine, Seoul, Korea.
}

Correspondence: J-H. Yoon, Dept of Otorhinolaryngology, Yonsei University College of Medicine, 134 Shinchon-dong, Seodaemun-gu, Seoul, 120-752, Korea. E-mail: jhyoondyuhs.ac

ABSTRACT The proprotein convertases (PCs) are serine proteases responsible for the proteolytic maturation of many precursor proteins involved in upper airway remodelling during nasal polyposis. We have previously found that PC1/3 is expressed in human nasal mucosa.

However, whether PC1/3 is related to nasal polyp formation has not been investigated. To gain insight into the functional role of PC1/3 in nasal polyps, we determined $\mathrm{PC} 1 / 3$ expression in nasal polyps by immunostaining, Western blotting and enzyme assays and generated stable cells expressing PC1/3 using airway epithelial cell line NCI-H292.

Nasal polyps exhibit increased PC1/3 expression compared to normal nasal mucosa. PC1/3 was expressed in neuroendocrine cells in normal nasal mucosa and it was also expressed in goblet and ciliated cells in nasal polyps. NCI-H292 cells stably expressing PC1/3 displayed morphological changes, enhanced cell proliferation and migration, downregulation of E-cadherin and cytokeratins and upregulation of $\mathrm{N}$-cadherin, vimentin, matrix metalloproteinase-2, collagen-I, snail and twist. Importantly, $\mathrm{PC} 1 / 3$ expression was positively correlated with epithelial-mesenchymal transition in cultured human nasal epithelial cells and in nasal polyps.

Taken together, our data suggest that $\mathrm{PC} 1 / 3$ overexpression induces morphological and phenotypic epithelial-mesenchymal transition changes of airway epithelial cells and these changes may contribute to the pathogenesis of nasal polyps.

@ERSpublications

PC1/3 overexpression induces morphological and phenotypic epithelial-mesenchymal transition changes in airway epithelium http://ow.ly/on9hZ

This article has supplementary material available from www.erj.ersjournals.com

Received: June 292012 | Accepted after revision: Jan 032013 | First published online: Jan 112013

Support statement: The Basic Science Research Programme of the National Research Foundation of Korea, which is funded by the Ministry of Education, Science and Technology, supported this work (grants 2012-0000803 (J-H. Yoon) and 2011-0026256 (S-N. Lee)).

Conflict of interest: None declared.

Copyright (OERS 2013 


\section{Introduction}

Chronic rhinosinusitis with nasal polyps (CRSwNP) is a chronic inflammatory sinus disease affecting $4 \%$ of people worldwide [1]. It is characterised by persistent inflammation and structural changes that are referred to as airway remodelling $[1,2]$. Airway remodelling in CRSwNP constitutes basement membrane thickening as a result of subepithelial fibrosis, increased deposition of extracellular matrix (ECM) and stromal oedema [3]. CRSwNP are associated with other disorders such as asthma, cystic fibrosis, bronchiectasis and aspirin sensitivity [1]. However, our understating of the pathogenesis of these diseases is still limited.

Epithelial cells can undergo the epithelial-mesenchymal transition (EMT) in response to tissue injury and inflammation, leading to organ fibrosis and cancer [2,4]. During EMT, epithelial cells lose their defining characteristics, such as E-cadherin and apicobasal polarity, and gain mesenchymal features, including upregulation of $\alpha$-smooth muscle actin (SMA), vimentin, matrix metalloproteinases (MMPs) and the transcription factors snail, slug and twist $[2,4,5]$. There is increasing evidence that lung epithelial injury and abnormal epithelial repair responses induce the persistent epithelial activation leading to pathological processes associated with tissue fibrosis by undergoing EMT [6, 7]. Recently, it has been shown that $\alpha$-SMA is expressed in epithelial cell lining in nasal polyps and hypoxia-induced EMT via a SMAD (mothers against decapentaplegic homologue)-3-dependent mechanism in cultured human nasal epithelial cells [8], indicating an important role for EMT in the pathogenesis of nasal polyps.

Many proteins, such as transcription growth factor (TGF)- $\beta$, vascular endothelial growth factor (VEGF), insulin-like growth factor (IGF)-1, epithelial growth factor, fibroblast growth factor-2, and MMPs can induce EMT [9]. Interestingly, these proteins are synthesised as inactive precursor proproteins, which require prodomain removal by proprotein convertases (PCs) to become a biologically active form [10]. PCs are a family of calcium-dependent subtilisin-like endoproteases, which include furin, PC1/3, PC2, PC4, paired basic amino acid cleaving enzyme (PACE)-4, PC5/6, PC7/lymphoma proprotein convertase/PC8 and mammalian subtilisin/kexin isozyme (SKI)-1/S1P [10]. Since PCs are responsible for the activation of protein precursors implicated in cancer and associated metastatic processes, they may be promising targets for therapy of different proliferative diseases. Indeed, early studies reported the elevated expression of PCs in various human cancers such as lung [11], breast [12] and endocrine-related cancers [13].

We have previously shown that $\mathrm{PC} 1 / 3$ is expressed in human nasal mucosa [14]. However, whether PC1/3 is related to nasal polyp formation has not been investigated. To gain insight into the functional role of PC1/3 in the nasal polyp epithelium, we determined PC1/3 expression in nasal polyps and generated stable cell lines constitutively expressing PC1/3 using NCI-H292 cells. Based on in vitro assays and proteomic analysis, we report that PC1/3 can induce the EMT-like process in airway epithelial cells.

\section{Materials and methods}

Human subjects

Inferior turbinate mucosa (normal nasal mucosa) specimens were obtained from six patients who had undergone septoplasties. Six nasal polyp specimens were obtained from patients with chronic rhinosinusitis who had no clinical history of asthma, aspirin sensitivity or cystic fibrosis. None of the patients had been on a regimen of intranasal medication, oral steroids or antibiotic treatment for 3 months prior to the study, and an allergic skin-prick test was negative for all of the patients.

\section{Cell culture and transfection}

The institutional review board of Yonsei University College of Medicine provided approval for this study (IRB\# 4-2012-0136). Normal human nasal epithelial (NHNE) cells were obtained from patients after surgical turbinectomies. The air-liquid interface culture system was used for NHNE cells (online supplementary material). To establish stable PC1/3-expressing cells, NCI-H292 cells were transfected with RcCMV encoding mouse PC1/3 with FuGENE ${ }^{\mathrm{TM}} 6$ (Roche, Indianapolis, IN, USA) (online supplementary material).

\section{RNA isolation, real-time PCR, and semi-quantitative reverse transcriptase-PCR}

Total RNA was isolated using TRIzol reagent (Invitrogen, Gaithersburg, MD, USA) according to the manufacturer's protocol. The cDNA was synthesised and amplified by PCR using specific primers for the indicated genes (table 1 and online supplementary material).

\section{Western blotting}

Specimens were ground to a fine powder in liquid nitrogen with a mortar and pestle. Ground tissues and NCI-H292 cells were homogenised in cell lysis buffer (Invitrogen, Camarillo, CA, USA) containing protease/phosphatase inhibitor cocktail on ice and subjected to $8 \%$ sodium dodecyl sulfate polyacrylamide 
TABLE 1 Primer sequences for real-time PCR

\begin{tabular}{|c|c|c|}
\hline Gene name & Forward primer $5^{\prime}-3^{\prime}$ & Reverse primer $5^{\prime}-3^{\prime}$ \\
\hline MMP-1 & AGTGACTGGGAAACCAGATGCTGA & GCTCTTGGCAAATCTGGCCTGTAA \\
\hline MMP-2 & ACATCAAGGGCATTCAGGAG & GCCTCCGTATACCGCATCAAT \\
\hline MMP-3 & ATTCCATGGAGCCAGGCTTTC & CATTTGGGTCAAACTCCAACTGTG \\
\hline MMP-7 & TGAGCTACAGTGGGAACAGG & TCATCGAAGTGAGCATCTCC \\
\hline MMP-8 & GAGGACAGAAAGAAAGCCAGGAG & AACTTTTCCAGGTAGTCCTGAA \\
\hline MMP-9 & TTGACAGCGACAAGAAGTGG & GCCATTCACGTCGTCCTTAT \\
\hline MMP-10 & CATACCCTGGGTTTTCCTCCAA & GTCCGCTGCAAAGAAGTATGTTTTC \\
\hline MMP-11 & CTAAAGGTATGGAGCGATGTGAC & GTCCAGGTCTCATCATAGTCGAA \\
\hline MMP-13 & TCCCAGGAATTGGTGATAAAGTAGA & CTGGCATGACGCGAACAATA \\
\hline MMP-14 & GAAGCCTGGCTACAGCAATATG & TGCAAGCCGTAAAACTTCTGC \\
\hline Alpha-5-integrin & АCTCAACTGCACCACCAATC & CCATCCATGAAGAGGGTATG \\
\hline Collagen I & TTGTGCGATGACGTGATCTGT & TTGGTCGGTGGGTGACTCTG \\
\hline Collagen IV & GCAAACGCTTACAGCTTTTGG & GGACGGCGTAGGCTTCTTG \\
\hline Fibronectin & CAGGATCACTTACGGAGAAACAG & GCCAGTGACAGCATACACAGTG \\
\hline GAPDH & GAAGGTGAAGGTCGGAGTC & GAAGATGGTGATGGGATTTC \\
\hline
\end{tabular}

gel electrophoresis (SDS-PAGE) followed by Western blotting using the appropriate antisera (online supplementary material).

Immunohistochemistry and immunofluorescence staining

PC1/3 was immunostained in formalin-fixed paraffin sections $(4 \mu \mathrm{m})$ of both normal nasal mucosa and nasal polyp specimens. For immunofluorescence staining, cyto-spin slides were prepared from epithelial cells of both normal nasal mucosa and nasal polyp specimens (online supplementary material).

\section{Enzyme assays}

Normal nasal mucosa and nasal polyps were briefly sonicated in $100 \mathrm{mM}$ sodium acetate, pH 5.5, $1 \%$ Triton X-100 and protease inhibitor cocktail. After centrifugation for $2 \mathrm{~min}$ at $12000 \times \mathrm{g}$, the supernatants were used for PC enzyme assays (online supplementary material).

In vitro wound assay

NCI-H292 cells were grown to confluence on a collagen I-coated 12-well culture plate. Cells were incubated with $5 \mu \mathrm{g} \cdot \mathrm{mL}^{-1}$ mitomycin C (Sigma Chemicals, Saint Louis, MO, USA) to block cell proliferation for $3 \mathrm{~h}$. An in vitro circular denuded area was made at the centre of the wells using a sterile pipette tip and then free cells were removed by PBS washing. At this time point $(\mathrm{t}=0 \mathrm{~h})$, wound margins were photographed. Then, the same fields of the wound margin were photographed at different time points. Cell morphology was imaged using a camera mounted on a microscope and processed using Adobe Photoshop software.

\section{Cell proliferation assay}

Cell growth was determined using the standard colorimetric cell counting kit-8 (Dojindo Laboratories, Kumamoto, Japan) according to the manufacturer's protocol. The absorbance at $450 \mathrm{~nm}$ was measured every 24 h over a 3-day period.

\section{Gelatin zymography}

Zymography was used to detect MMP-2 activity that was secreted into conditioned media (online supplementary material).

\section{Statistical analysis}

Data are expressed as the mean \pm SD of three experiments. Differences in value distribution were statistically validated using one-way ANOVA. Statistical analysis was performed using the program GraphPad Prism 3 for Windows (GraphPad Software, Inc., La Jolla, CA, USA). Differences were considered to be significant at $\mathrm{p}<0.05$.

\section{Results}

PC1/3 is expressed in goblet and ciliated cells as well as neuroendocrine cells in the nasal polyp epithelium

We first examined PC1/3 distribution in both normal nasal mucosa and nasal polyps by immunohistochemical staining. Figure 1a shows that PC1/3 immunoreactivity was restricted to some specialised cells 

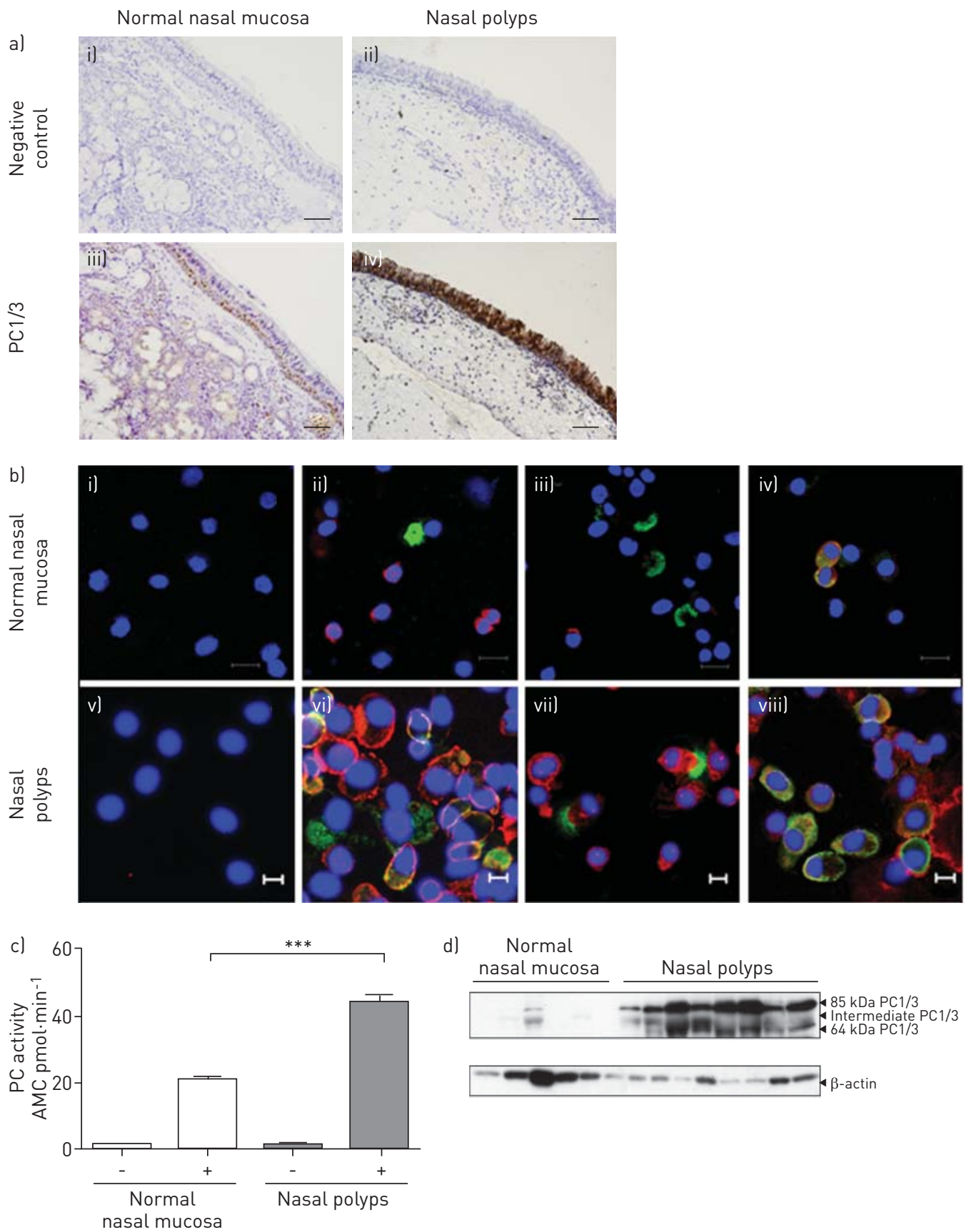

d) Normal
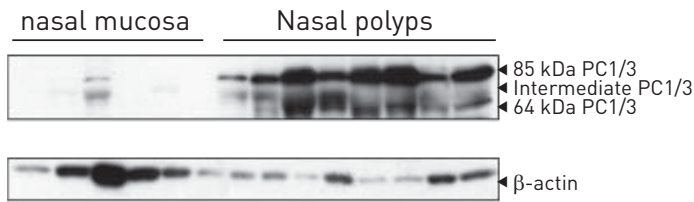

FIGURE 1 Localisation of proprotein convertase (PC) $1 / 3$ in normal human nasal mucosa and nasal polyps. a) PC1/3 localisation was analysed by immunohistochemical staining with antiserum against PC1/3. The positive signal of PC1/3 was restricted to some specialised basal cells in normal nasal mucosa (iii), while nasal polyps showed intense $\mathrm{PC1} / 3$ immunoreactivity in all epithelial cells (iv). An IgG control is shown (i, ii). The experiment was repeated using four normal nasal mucosa and four nasal polyp specimens. Scale bars $=100 \mu \mathrm{m}$. b) Localisation of PC1/3 both in normal nasal mucosa and nasal polyps was analysed by immunostaining with either antisera against PC1/3 and mucin (MUC)-5AC (ii, vi), antisera against $\mathrm{PC} 1 / 3$ and acetylated tubulin (iii, vii) or antisera against $\mathrm{PC} 1 / 3$ and chromogranin $\mathrm{A}(\mathrm{Chr} \mathrm{A})$ (iv, viii) using cytospin slides prepared from epithelial cells of both normal nasal mucosa (i-iv) and nasal polyps ( $v$-viii). Nuclei were stained with 4',6-diamidino-2-phenylindole (DAPI). An IgG control is shown (i, v). i-iv) scale bars=100 $\mu \mathrm{m}$; v-viii) scale bars $=10 \mu \mathrm{m} . \mathrm{c})$ Tissue extracts $(100 \mu \mathrm{g})$ obtained from both normal nasal mucosa and nasal polyps were used to determine total intracellular PC activity. Data represent $n=6$ tissues per group, each measured in triplicate, mean \pm SD. AMC: 7-amido4-methylcoumarin. ${ }^{* * *}: \mathrm{p}<0.001$ by one-way ANOVA. d) Expression of PC1/3 was analysed by Western blot of crude extracts $(50 \mu \mathrm{g})$ prepared from both normal nasal mucosa and nasal polyps. $\beta$-actin was used as a loading control. 
a)

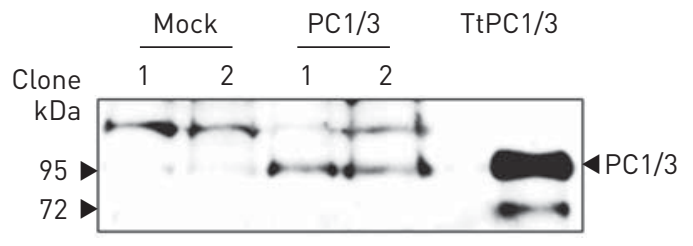

$\beta$-actin

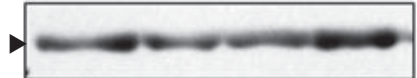

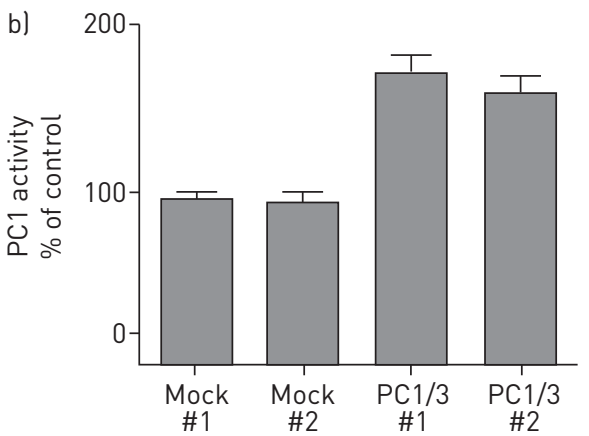

c)
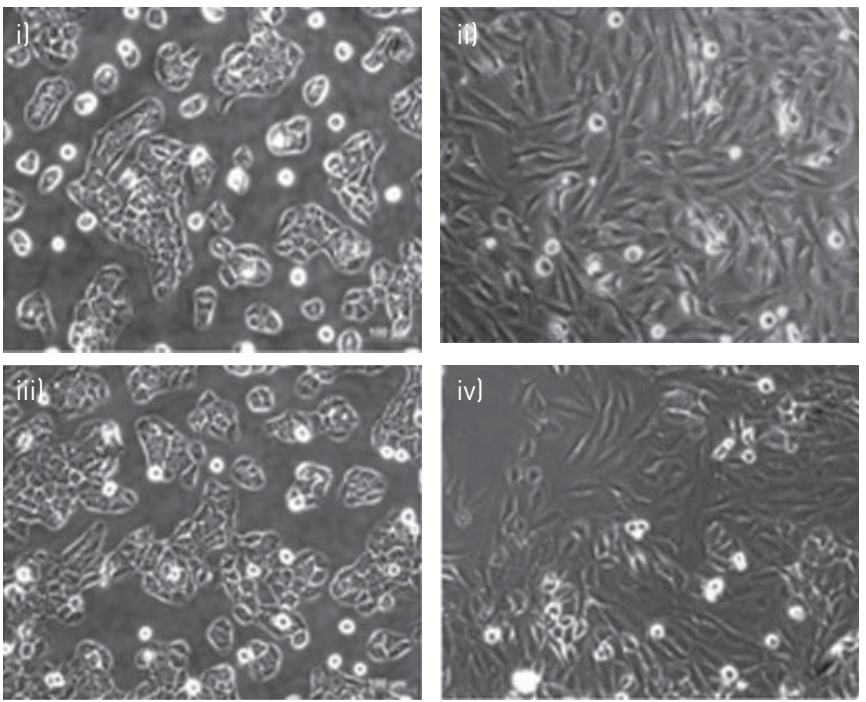

FIGURE 2 Proprotein convertase (PC) 1/3 overexpression induces morphological changes in NCI-H292 cells. a) NCI$\mathrm{H} 292$ cells were stably transfected with the expression plasmid containing $\mathrm{mPC} 1 / 3 \mathrm{cDNA}$ and $\mathrm{PC} 1 / 3$ production was analysed by Western blot. An immunoreactive product with a molecular weight of $87 \mathrm{kDa}$ corresponding to the predicted molecular size for PC1/3 was detected in PC1/3 cells. "Mock" represents a stable cell line transfected with an empty vector and "PC1/3" represents stable cell lines expressing PC1/3; two independent high-expressing cells were used for the experiments. NCI-H292 cells transiently expressing PC1/3 (TtPC1/3) were used as a positive control. b) NCI-H292 cells were cultured in a 12-well plate overnight. The medium was changed to Opti-MEM containing $100 \mu \mathrm{g} \cdot \mathrm{mL}^{-1}$ aprotinin and collected after 16 h. 50- $\mu$ L medium samples were assayed for PC1/3 activity. c) i) and iii) mock cells showed a flat and cobblestone shape with tight junctions, whereas ii), iv) PC1/3 cells displayed a loss of cell-cell contacts and adopted a more spindly elongated shape.

located at the base of the epithelium in normal nasal mucosa (fig. 1a(iii)), whereas in nasal polyps, PC1/3 was strongly detected in the entire epithelial cell layer (fig. la(iv)). No staining was detected with an IgG control (fig. 1a(i, ii)).

To examine which cell types in both normal nasal mucosa and nasal polyps express PC1/3, cytospin slides prepared from epithelial cells of both normal nasal mucosa and nasal polyps were subjected to double immunocytofluorescence staining using either antisera against PC1/3 and mucin (MUC)-5AC (a goblet cell marker), antisera against PC1/3 and acetylated tubulin (a ciliated cell marker) or antisera against PC1/3 and chromogranin A (ChrA, a secretory granule marker) [15]. Consistent with the immunohistochemical results, PC1/3 was not colocalised with both MUC5AC (fig. 1b(ii)) and acetylated tubulin (fig. 1b(iii)) in normal nasal mucosa. In contrast, in nasal polyps, some cells were positively labelled for both $\mathrm{PC} 1 / 3$ and MUC5AC (fig. 1b(vi): yellow) and others were labelled solely for either PC1/3 (fig. 1b(vi): red) or MUC5AC (fig. $1 \mathrm{~b}$ (vi): green), indicating that PC1/3 is expressed in goblet cells. In addition, PC1/3 was also present in ciliated cells as evidenced by colocalisation with acetylated tubulin in nasal polyps (fig. $1 \mathrm{~b}$ (vii)). In both normal nasal mucosa and nasal polyps, $\mathrm{PC1} / 3$ was located in ChrA-containing secretory granules of neuroendocrine cells, seen as yellow objects in the merged image (fig. $1 \mathrm{~b}$ (iv, viii). No staining was detected in an IgG control (fig. $1 \mathrm{~b}(\mathrm{i})$ ).

We next explored whether the storage of active PCs in nasal polyps differs from normal nasal mucosa by measuring total PC enzymatic activity. Total enzyme activity of PCs was significantly higher in nasal polyps 

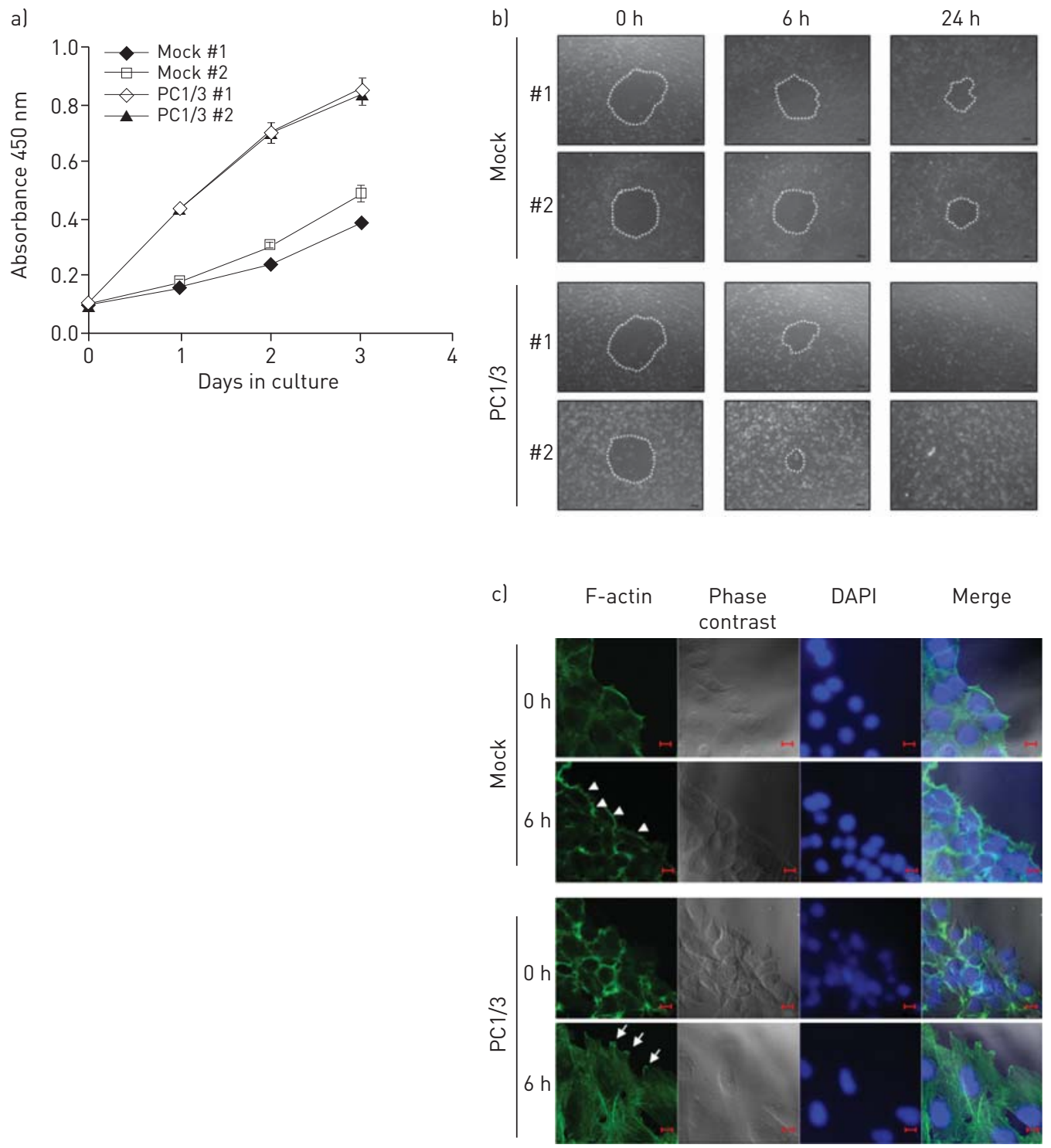

FIGURE 3 Proprotein convertase (PC)1/3 overexpression facilitates cell proliferation and migration in NCI-H292 cells. a) Cell proliferation was quantified at 1,2 and 3 days using a cell counting kit- 8 assay kit (Dojindo Laboratories, Kumamoto, Japan). Data are expressed as the mean \pm SD of three individual experiments, each measured in triplicate. b) Cells were grown to confluence on a collagen I-coated 12-well culture plate. Cells were treated with mitomycin C $\left(5 \mu \mathrm{g} \cdot \mathrm{mL}^{-1}\right)$ to block cell proliferation. The wound area was measured 6 or $24 \mathrm{~h}$ after wounding. The wound margin is depicted by a white dotted line. c) Cells were stained for filamentous (F)-actin using fluorescein isothiocyanate (FITC)phalloidin. Mock cells exhibited a typical stationary phenotype with marginal actin bundles (arrowheads) $6 \mathrm{~h}$ after wounding, whereas PC1/3 cells showed the formation of extensive actin stress fibres throughout the cells and filopodial extension (arrows) at the leading edge of a wound. DAPI: 4',6-diamidino-2-phenylindole. Scale bars $=10 \mu \mathrm{m}$.

compared to normal nasal mucosa (fig. 1c). In addition, Western blotting showed that PC1/3 expression was considerably increased in nasal polyps (fig. 1d). Figure 1d displays three PC1/3 species with molecular weights of $\sim 64 \mathrm{kDa}$ (C-terminally truncated form), $71 \mathrm{kDa}$ (intermediate form with a cleavage at an internal C-terminal site) and $85 \mathrm{kDa}$ (mature form), as $\mathrm{PC1} / 3$ undergoes proteolytic cleavage during the activation process. These data suggest that $\mathrm{PC} 1 / 3$ may be related to nasal polyp formation.

\section{PC1/3-overproducing $\mathrm{NCl}-\mathrm{H} 292$ cells exhibit morphological changes}

To address the functional role of PC1/3 in airway epithelial cells, we generated stable cell lines constitutively producing PC1/3 using NCI-H292 cells. Cells were stably transfected with either RcCMV vector containing PC1/3 cDNA (designated as PC1/3 cells; two independent cells: 1 and 2) or RcCMV alone as a control (mock cells: 1 and 2). Western blot analysis showed an immunoreactive band with a molecular weight of $85 \mathrm{kDa}$ corresponding to the mature form of PC1/3 (fig. 2a, PC1/3); a transiently transfected clone with 
FIGURE 4 Proprotein convertase (PC) $1 / 3$ overexpression induces upregulation of epithelial-mesenchymal transitionrelated protein production in NCIH292 cells. PC1/3 cells exhibited dramatically decreased expression of E-cadherin and cytokeratin- 8 and -18 , and strongly increased expression of $\mathrm{N}$-cadherin, vimentin, snail and twist.
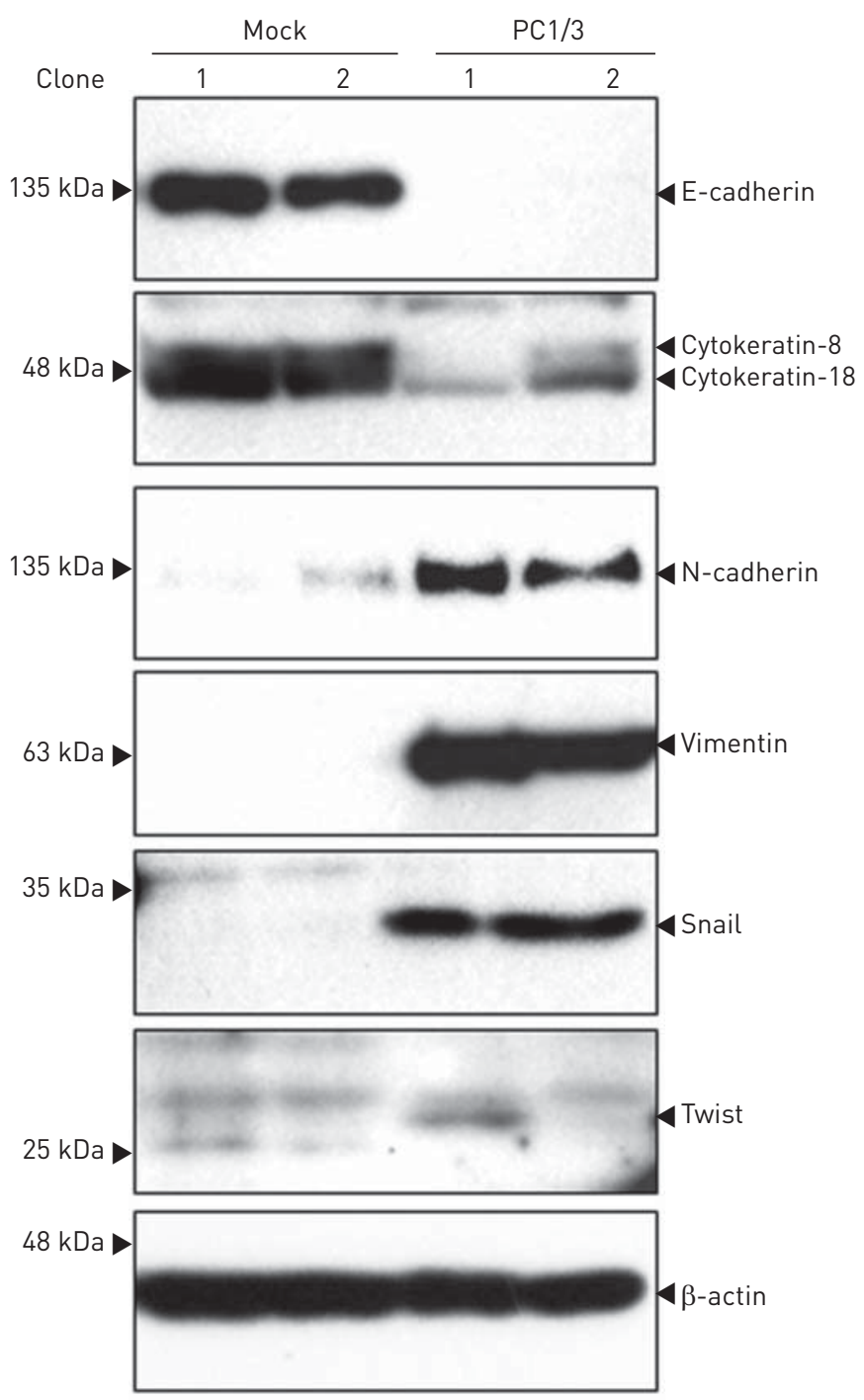

PC1/3 cDNA was used as a positive control (TtPC1/3). We confirmed this result by measuring PC1/3 activity using conditioned media (fig. 2b). Interestingly, mock cells exhibited a cobblestone-like shape with cell-cell junctions (fig. 2c(i, iii)), while PC1/3 cells showed an elongated and spindled shape, losing the cellcell contacts (fig. $2 \mathrm{c}(\mathrm{ii}, \mathrm{iv})$ ).

\section{PC1/3 overexpression modulates cell growth and migration in $\mathrm{NCl}-\mathrm{H} 292$ cells}

We next evaluated cell proliferation and migration in mock and $\mathrm{PC} 1 / 3$ cells. Over a 3 -day period, $\mathrm{PC} 1 / 3$ cells showed significantly enhanced cell proliferation as compared to mock cells (fig. $3 \mathrm{a}$ ). To assess the migratory activity, we performed the migration assay in the presence of $5 \mu \mathrm{g} \cdot \mathrm{mL}^{-1}$ mitomycin $\mathrm{C}$ to inhibit cell proliferation and to confirm that wound healing was attributed to cell migration. The wound area was measured 6 or $24 \mathrm{~h}$ after wounding. Figure $3 \mathrm{~b}$ shows that the wound area decreased in a time-dependent manner. Over a 24 -h period, mock cells recovered approximately $44 \%$ of the wound area, whereas $\mathrm{PC} 1 / 3$ cells completely recovered the wound area, indicating that PC1/3 cells gained a migratory phenotype.

Migrating cells generally display a plasma membrane protrusion associated with actin reorganisation at the leading edge [16]. We further analysed cell shape at the wound edge and the distribution of filamentous actin by fluorescein isothiocyanate-phalloidin staining (fig. 3c). After 6 h, mock cells displayed the formation of marginal actin bundles at the edge of the cells, which is a typical stationary phenotype. In contrast, PC1/3 cells showed an elongated spreading shape, the formation of extensive actin stress fibres throughout the cells and lamellipodial and filopodial extensions at the leading edge of the wound. 
a)

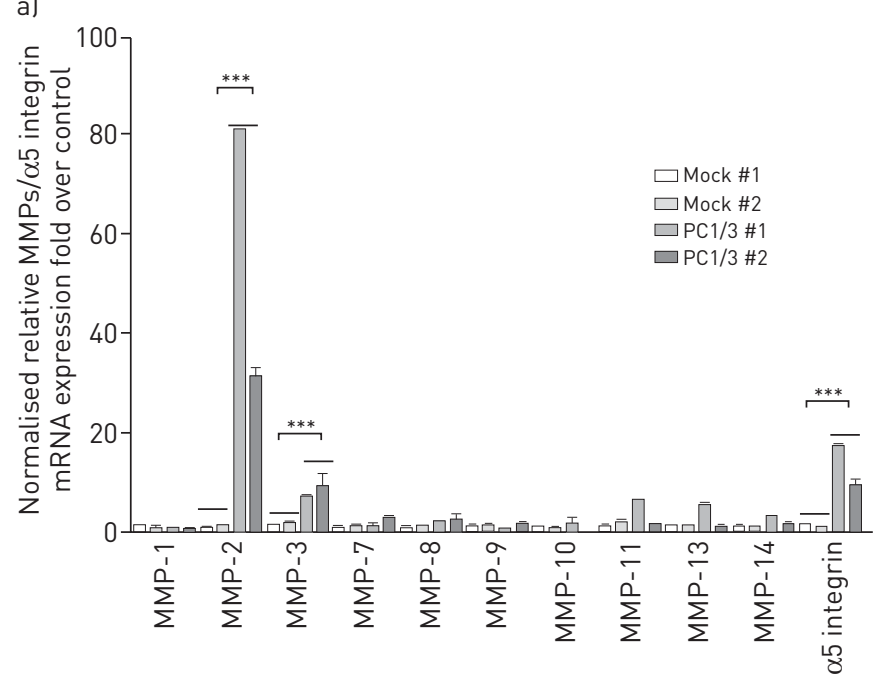

c)

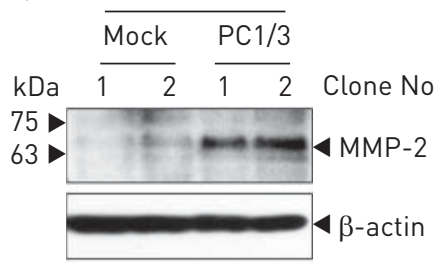

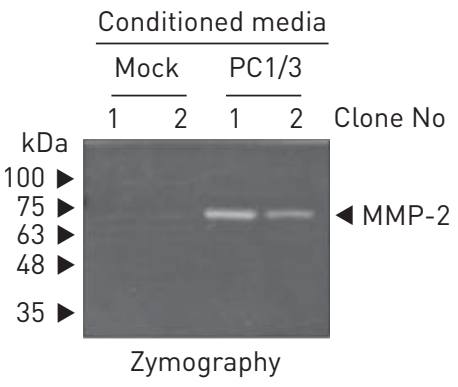

b)

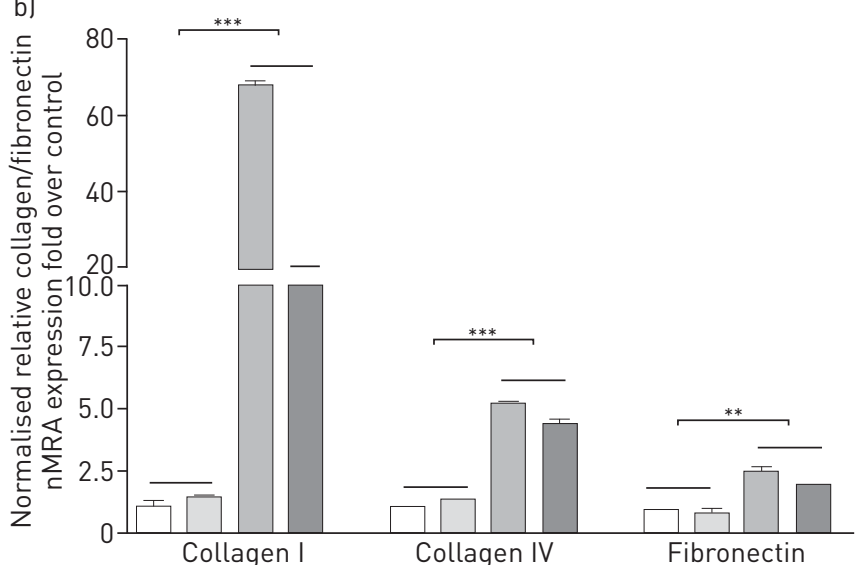

d)

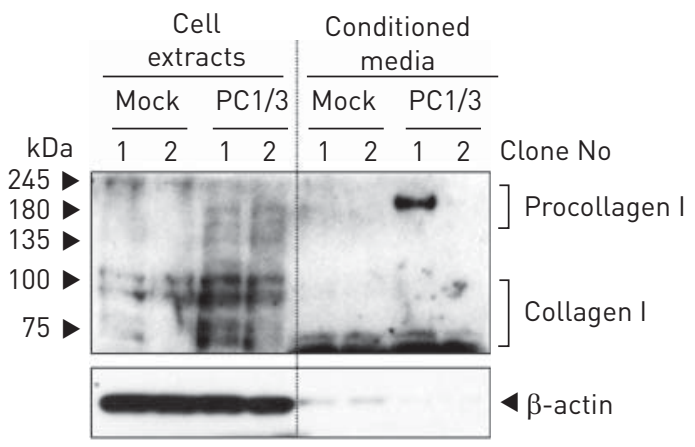

FIGURE 5 Proprotein convrtase (PC) 1/3 overexpression upregulates expression of matrix metalloproteinases (MMPs) and extracellular matrix proteins in NCI$\mathrm{H} 292$ cells. a) Real-time quantitative reverse transcriptase (RT)-PCR analysis showed significantly increased gene expression of MMPs and $\alpha 5$-integrin in PC1/3 cells compared to mock cells. Data are presented as the mean \pm SD of three individual experiments, each measured in triplicate. ${ }^{* * *}$ : $p<0.001$ by one-way ANOVA. b) Expression of extracellular matrix genes collagen I, collagen IV and fibronectin was evaluated using real-time quantitative RT-PCR. Data are presented as the mean \pm SD of three individual experiments, each measured in triplicate. ${ }^{* *}: \mathrm{p}<0.01 ;{ }^{* *}: \mathrm{p}<0.001$, both by one-way ANOVA. c) MMP-2 expression and activity is upregulated in PC1/3 cells, as demonstrated by Western blot analysis of cell extracts (left panel) and zymographic analysis of conditioned media (right panel). d) The increase of collagen I expression was shown by Western blot analysis of cell extracts and conditioned media.

Collectively, our results suggest that $\mathrm{PC} 1 / 3$ overexpression can facilitate cell proliferation and induces a migratory phenotype in NCI-H292 cells.

\section{PC1/3 overexpression enhances expression of EMT markers, MMPs and ECM proteins in $\mathrm{NCl}-\mathrm{H} 292$} cells

The expression of EMT markers was analysed by Western blotting. PC1/3 cells exhibited downregulation of the epithelial markers E-cadherin and cytokeratin-8 and -18, and upregulation of the mesenchymal markers $\mathrm{N}$-cadherin, vimentin, snail and twist, when compared to mock cells (fig. 4).

Since the upregulation of MMPs, $\alpha 5$-integrin and the ECM proteins collagen and fibronectin are common features of EMT [17-20], we assessed the expression levels of these genes in mock and PC1/3 cells using realtime PCR. Figure 5a shows that PC1/3 overexpression resulted in markedly enhanced expression of MMP-2, MMP-3, and $\alpha 5$-integrin in NCI-H292 cells, while the expression levels of MMP-1, -7, -9 and -10 were not changed; increased MMP-2 expression was confirmed by Western blotting and zymography (fig. 5b). We also observed a dramatic upregulation of collagen I together with increased expression of collagen IV and fibronectin (fig. 5c); collagen I upregulation was also confirmed by Western blotting (fig. 5d). Taken together, these data indicate that PC1/3 can induce EMT-like phenotypes in NCI-H292 cells.

PC1/3 expression is positively correlated with EMT, both in cultured NHNE cells and in nasal polyps We next examined whether PC1/3 expression is altered during EMT induced by TGF- $\beta 1$ and cytomix (a combination of tumour necrosis factor (TNF)- $\alpha$ and interleukin (IL)-1 $\beta$ ) in NHNE cells; TGF- $\beta 1$, TNF- $\alpha$ and IL- $1 \beta$ are able to induce EMT in vitro $[6,7]$. Figure 6 a shows that TGF- $\beta 1$ and cytomix suppressed the 
a)
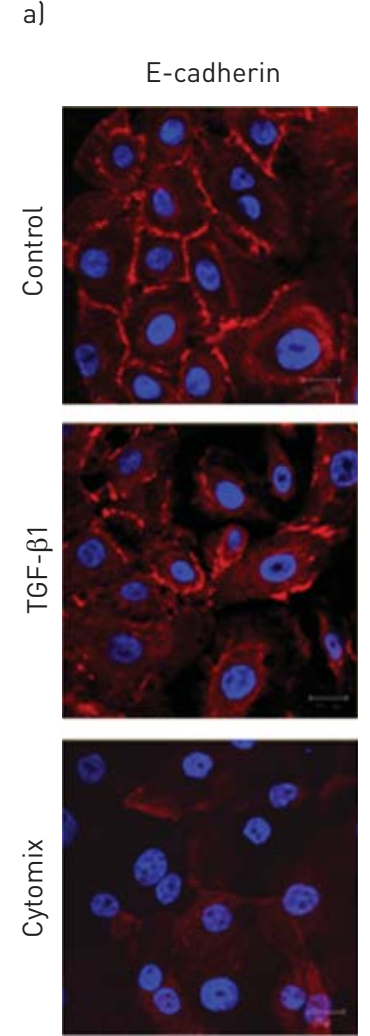

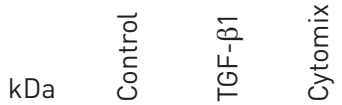
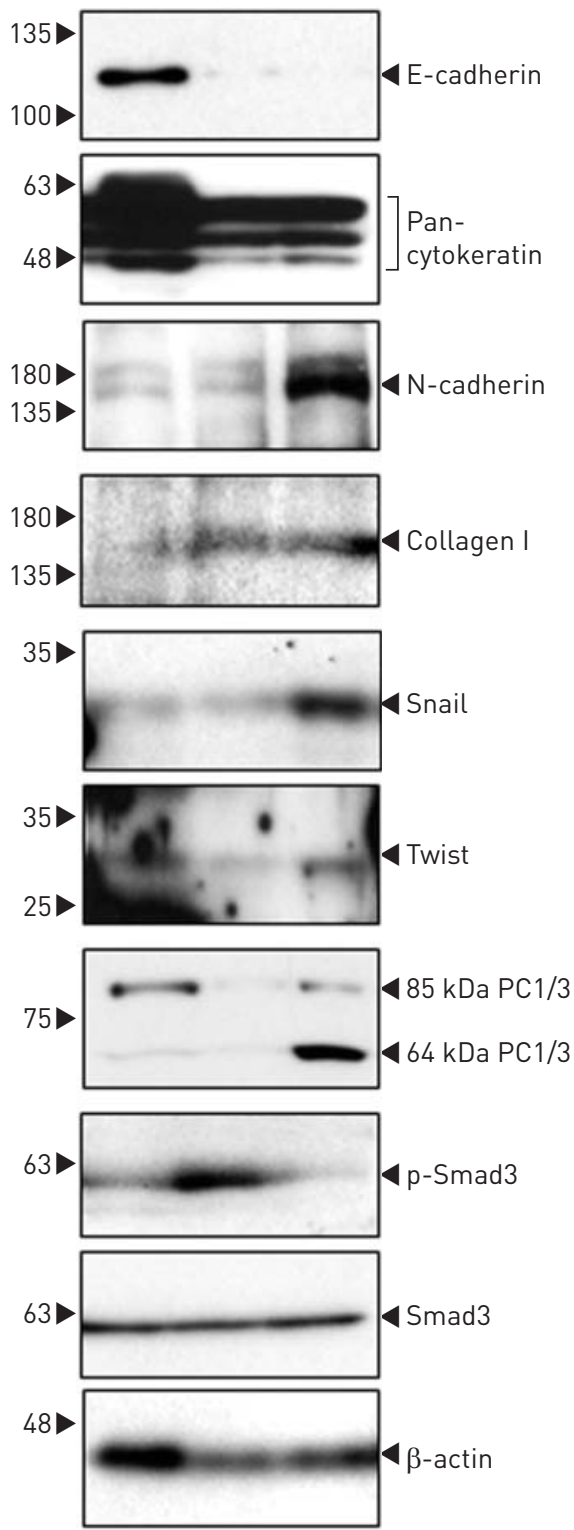

b)

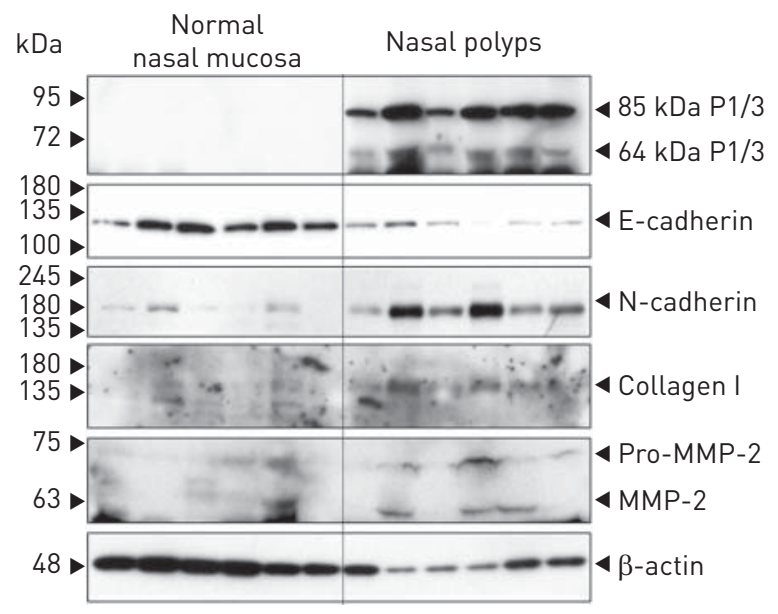

FIGURE 6 Proprotein convertase (PC) $1 / 3$ is upregulated in both cultured normal human nasal epithelial cells and nasal polyps that undergo epithelialmesenchymal transition. a) Cultured normal human nasal epithelial (NHNE) cells were incubated with either $10 \mathrm{ng} \cdot \mathrm{mL}^{-1}$ transforming growth factor (TGF)- $\beta 1$ or a combination of $5 \mathrm{ng} \cdot \mathrm{mL}^{-1}$ tumour necrosis factor- $\alpha$ and $5 \mathrm{ng} \cdot \mathrm{mL}^{-1}$ interleukin-1 $\beta$ (cytomix) for $72 \mathrm{~h}$. NHNE cells were fixed for immunocytofluorescence staining of E-cadherin (left panel) or cell extracts were taken for Western blotting (right panel). Both TGF- $\beta 1$ and cytomix changes the expression of epithelialmesenchymal transition (EMT) markers and collagen I, while PC1/3 expression was only elevated by cytomix. p: phospho; SMAD: mothers against decapentaplegic homologue. Scale bars $=20 \mu \mathrm{m}$. b) Tissue extracts $(50 \mu \mathrm{g})$ obtained from both normal nasal mucosa and nasal polyps were used to determine the expression changes of EMT markers, collagen I and PC1/3. MMP: matrix metalloproteinase. $\beta$-actin was used as a loading control in both instances.

expression of E-cadherin and pan-cytokeratin and increased the expression of several mesenchymal markers $\mathrm{N}$-cadherin, collagen I, snail and twist. Interestingly, the expression levels of N-cadherin, snail and twist were significantly higher in cytomix-treated NHNE cells compared to TGF- $\beta 1$-treated cells. Moreover, PC1/ 3 expression was induced by cytomix, but not by TGF- $\beta 1$, indicating the involvement of PC1/3 in cytomixmediated EMT, but not in TGF- $\beta 1$-induced EMT; TGF- $\beta 1$ signalling was examined by measuring the levels of phospho-SMAD3 (p-SMAD3) [8]. We further examined the relationships between PC1/3 and EMT markers in nasal polyps and found that $\mathrm{PC} 1 / 3$ expression was positively correlated with loss of E-cadherin 
expression and gain in expression of N-cadherin, collagen I and MMP-2 (fig. 6b). Collectively, these results suggest that PC1/3 may play an important role in nasal polyps undergoing EMT.

\section{Discussion}

Inflammatory and remodelling processes of the upper airway mucosa are believed to play important roles in the development of nasal polyps [3]. Many proteins implicated in remodelling, such as growth factors and their receptors and MMPs [9] should be proteolytically cleaved into biologically active forms by PCs [10]. We report here that $\mathrm{PC} 1 / 3$ expression is strongly increased in nasal polyps compared with normal nasal mucosa and induces the EMT-like process in airway epithelial cells. We also note that total PC activity was significantly higher in nasal polyps compared to normal nasal mucosa; the convertases furin, PC5/6 and PACE4 have a broad $\mathrm{pH}$ optimum with peak activity at neutral to weakly basic $\mathrm{pHs}$ (6.0-8.5 for furin, 7.0-8.0 for PC5/6 and 7.0-8.5 for PACE4), while PC1/3 is active within a much narrower $\mathrm{pH}$ range (5.0-6.5) [21]. Therefore, we speculate that a large portion of this enzyme activity under our PC enzyme assay conditions ( $\mathrm{pH}$ 5.5) is originated from endogenous $\mathrm{PC} 1 / 3$.

We demonstrate that $\mathrm{PC} 1 / 3$ is expressed in ChrA-containing secretory granules in neuroendocrine cells located at the base of the nasal epithelium. PC1/3 is known to be predominantly expressed in neural and endocrine cells and localised in secretory granules [22]. It has been reported that PC1/3 and its physiological substrates, such as calcitonin gene-related peptide, leu-enkephalin and bombesin, are expressed in neuroendocrine cells scattered in the respiratory epithelium [23]. Furthermore, JORNOT et al. [15] reported that primary human nasal epithelial cells contain brain-derived neurotrophic factor, a PC1/3 substrate [24], in ChrA-containing secretory granules in neuroendocrine cells. Surprisingly, PC1/3 is also expressed in goblet and ciliated cells in the nasal polyp epithelium. CAROLAN et al. [25] have shown that ubiquitin carboxyl-terminal hydrolase (UCH)-L1, a marker of neuroendocrine cells, is expressed only in neuroendocrine cells of the airway epithelium in nonsmokers, while in smokers UCH-L1 is also upregulated in ciliated epithelial cells. In line with these studies, we suggest that both goblet cells and ciliated cells can transdifferentiate to other epithelial cells. Collectively, we hypothesise that PC1/3 may have an important role in the pathogenesis of nasal polyps through its ability to produce a variety of active peptides.

In this study, we investigated the biological consequences of PC1/3 overexpression in NCI-H292 cells (PC1/3 cells); NCI-H292 cells maintain mucoepidermoid structure in culture and readily accept gene transfer. Surprisingly, PC1/3 cells displayed a morphological transformation from typical epithelial-like shape to an elongated and spindled morphology. This result matches a previous finding that PC1/3-overexpressing Michigan Cancer Foundation (MCF)-7 cells, a human breast cancer cell line, exhibit morphological changes with elongated cell processes, possibly due to alterations in cell adhesion molecule processing such as integrins, fibronectin and collagens by PC1/3 [26]. We also demonstrate that PC1/3 overexpression substantially increased cell proliferation. Since growth factors such as IGF-1, VEGF and platelet-derived growth factor play a critical role in tumour cell growth [27], it might be possible that these substrates are processed by PC1/3 in NCI-H292 cells, resulting in enhanced cell proliferation.

Cell migration is important in many physiological and pathological processes, such as normal development, tumour invasion, inflammatory responses and wound repair [28]. Cell migration is mediated by altered focal adhesion dynamics in combination with the protrusion of lamellipodia at the leading edge to generate forward cell movement [29]. We observed that PC1/3 cells increase the migration speed significantly after wounding and display more elongated and prominent perpendicular actin stress fibres as well as lamellipodial and filopodial extensions when compared to mock cells. In addition, PC1/3 cells display downregulation of epithelial markers and upregulation of mesenchymal markers, referred to as EMT $[2,4]$. Recently, SHIN et al. [8] showed hypoxia-induced EMT via a SMAD3-dependent mechanism in cultured human nasal epithelial cells and in epithelial cells lining nasal polyps, suggesting an important role for EMT in the pathogenesis of nasal polyps. Our proteomic analysis shows that differentially produced proteins between PC1/3 cells and mock cells are implicated in carbohydrate metabolism, cell proliferation, cytoskeletal reorganisation, DNA metabolism and signal transduction (online supplementary figure S1 and table S2). Interestingly, UCH-L1 expression is upregulated in $\mathrm{PC1} / 3$ cells (online supplementary figure S1c and table S2). As previously stated, $\mathrm{UCH}-\mathrm{L} 1$ is a neuroendocrine cell-specific protein used as a marker in assessing lung cancer [25].

During the repair process after airway epithelial injury, cells actively synthesise $\alpha 5$-integrin at the leading edge of cells $[19,20]$ and require a functional cooperation of integrins with MMP-14 for cell migration [17]. These proteins are PC substrates and activated MMP-14 can facilitate the maturation of pro-MMP-2 [17]. SEOMUN et al. [30] reported that MMP-2-overexpressing human lens epithelial cells show typical characteristics of a myofibroblast-like cell phenotype, indicating a role of MMP-2 in the pathological fibrosis. Here, we show that PC1/3 overexpression induces upregulation of MMP-2, MMP-14 and 
a5-integrin expression in NCI-H292 cells. MMP-3 levels were also increased in PC1/3 cells; MMP-3 production in mammary epithelial cells induces EMT [18].

We demonstrate that the expression of PC1/3 and twist are upregulated in NHNE cells undergoing EMT in response to TNF- $\alpha /$ IL-1 $\beta$. Li et al. [5] have reported that TNF- $\alpha$ and IL-1 $\beta$ induce EMT in both normal breast epithelial and breast cancer cells via necrosis factor- $\kappa \mathrm{B}$-mediated twist upregulation. We also show that $\mathrm{PC} 1 / 3$ expression is strongly correlated with EMT in nasal polyps.

In summary, our data provide insights into the plasticity of cellular phenotypes in the nasal polyp epithelium and possible novel functions of PC1/3 by inducing an EMT-like process in the pathogenesis of nasal polyps. Further studies are needed to identify the physiological substrates of PC1/3 and to advance the understanding of the role of $\mathrm{PC} 1 / 3$ in the pathogenesis of nasal polyps. Clinically, the development of specific inhibitors or modulators of $\mathrm{PC} 1 / 3$ may find relevant applications in the control of this pathology.

\section{Acknowledgements}

We thank Iris Lindberg (University of Maryland, Baltimore, MD, USA) for the RcCMV expression vector harbouring the mouse PC1/3 cDNA and antimouse PC1/3 antibody.

\section{References}

1 Guilemany J, Angrill J, Alobid I, et al. United airways: the impact of chronic rhinosinusitis and nasal polyps in bronchiectasic patient's quality of life. Allergy 2009; 64: 1524-1529.

2 Jeffery PK. Remodeling and inflammation of bronchi in asthma and chronic obstructive pulmonary disease. Proc Am Thorac Soc 2004; 1: 176-183.

3 Pawankar R, Nonaka M. Inflammatory mechanisms and remodeling in chronic rhinosinusitis and nasal polyps. Curr Allergy Asthma Rep 2007; 7: 202-208.

4 Kalluri R, Neilson EG. Epithelial-mesenchymal transition and its implications for fibrosis. J Clin Invest 2003; 112: $1776-1784$.

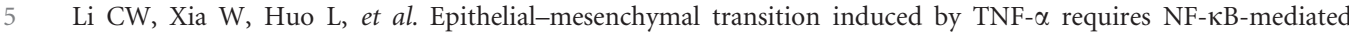
transcriptional upregulation of Twist1. Cancer Res 2012; 72: 1290-1300.

6 Câmara J, Jarai G. Epithelial-mesenchymal transition in primary human bronchial epithelial cells is Smaddependent and enhanced by fibronectin and TNF- $\alpha$. Fibrogenesis Tissue Repair 2010; 3: 2-13.

7 Davies DE. The role of the epithelium in airway remodeling in asthma. Proc Am Thorac Soc 2009; 6: 678-682.

8 Shin HW, Cho K, Kim DW, et al. Hypoxia-inducible factor 1 mediates nasal polypogenesis by inducing epithelialto-mesenchymal transition. Am J Respir Crit Care Med 2012; 185: 944-954.

9 Kalluri R, Weinberg RA. The basics of epithelial-mesenchymal transition. J Clin Invest 2009; 119: 1420-1428.

10 Khatib AM, Siegfried G, Chrétien M, et al. Proprotein convertases in tumor progression and malignancy: novel targets in cancer therapy. Am J Pathol 2002; 160: 1921-1935.

11 Mbikay M, Sirois F, Yao J, et al. Comparative analysis of expression of the proprotein convertases furin, PACE4, PC1 and PC2 in human lung tumours. Br J Cancer 1997; 75: 1509-1514.

12 Cheng M, Watson PH, Paterson JA, et al. Pro-protein convertase gene expression in human breast cancer. Int J Cancer 1997; 71: 966-971.

13 Takumi I, Steiner D, Sanno N, et al. Localization of prohormone convertases $1 / 3$ and 2 in the human pituitary gland and pituitary adenomas: analysis by immunohistochemistry, immunoelectron microscopy, and laser scanning microscopy. Mod Pathol 1998; 11: 232-238.

14 Lee S, Ryu J, Joo J, et al. $\alpha$-Melanocyte-stimulating hormone inhibits tumor necrosis factor $\alpha$-stimulated MUC5AC expression in human nasal epithelial cells. Am J Respir Cell Mol Biol 2011; 44: 716-724.

15 Jornot L, Lacroix J, Rochat T. Neuroendocrine cells of nasal mucosa are a cellular source of brain-derived neurotrophic factor. Eur Respir J 2008; 32: 769-774.

16 Condeelis J. How is actin polymerization nucleated in vivo? Trends Cell Biol 2001; 11: 288-293.

17 Stawowy P, Fleck E. Proprotein convertases furin and PC5: targeting atherosclerosis and restenosis at multiple levels. J Mol Med (Berl) 2005; 83: 865-875.

18 Lochter A, Galosy S, Muschler J, et al. Matrix metalloproteinase stromelysin-1 triggers a cascade of molecular alterations that leads to stable epithelial-to-mesenchymal conversion and a premalignant phenotype in mammary epithelial cells. J Cell Biol 1997; 139: 1861-1872.

19 Pilewski J, Latoche J, Arcasoy S, et al. Expression of integrin cell adhesion receptors during human airway epithelial repair in vivo. Am J Physiol 1997; 273: L256-L263.

20 Hérard A, Pierrot D, Hinnrasky J, et al. Fibronectin and its $\alpha 5-\beta 1$-integrin receptor are involved in the woundrepair process of airway epithelium. Am J Physiol 1996; 271: L726-L733.

21 Lee SN, Kacprzak MM, Day R, et al. Processing and trafficking of a prohormone convertase 2 active site mutant. Biochem Biophys Res Commun 2007; 355: 825-829.

22 Seidah N, Day R, Marcinkiewicz M, et al. Mammalian neural and endocrine pro-protein and pro-hormone convertases belonging to the subtilisin family of serine proteinases. Enzyme 1991; 45: 271-284.

23 Cutz E, Chan W, Track N. Bombesin, calcitonin and leu-enkephalin immunoreactivity in endocrine cells of human lung. Experientia 1981; 37: 765-767.

24 Marcinkiewicz M, Savaria D, Marcinkiewicz J. The pro-protein convertase PC1 is induced in the transected sciatic nerve and is present in cultured Schwann cells: comparison with PC5, furin and PC7, implication in pro-BDNF processing. Mol Res Mol Brain Res 1998; 59: 229-246.

25 Carolan BJ, Heguy A, Harvey BG, et al. Up-regulation of expression of the ubiquitin carboxyl-terminal hydrolase L1 gene in human airway epithelium of cigarette smokers. Cancer Res 2006; 66: 10729-10740.

26 Cheng $\mathrm{M}, \mathrm{Xu} \mathrm{N}$, Iwasiow B, et al. Elevated expression of proprotein convertases alters breast cancer cell growth in response to estrogen and tamoxifen. J Mol Endocrinol 2001; 26: 95-105. 
27 Bassi DE, Fu J, Lopez de Cicco R, et al. Proprotein convertases: "master switches" in the regulation of tumor growth and progression. Mol Carcinog 2005; 44: 151-161.

28 Ponti A, Machacek M, Gupton S, et al. Two distinct actin networks drive the protrusion of migrating cells. Science 2004; 305: 1782-1786.

29 Heasman SJ, Ridley AJ. Mammalian Rho GTPases: new insights into their functions from in vivo studies. Nat Rev Mol Cell Biol 2008; 9: 690-701.

30 Seomun Y, Kim J, Lee EH, et al. Overexpression of matrix metalloproteinase-2 mediates phenotypic transformation of lens epithelial cells. Biochem J 2001; 358: 41-48. 